\title{
Breaking Barriers to Care: A Community of Solution for Chronic Disease Management
}

\author{
Jim Sanders, MD, MPH, Bill Solberg, MSW, and Michael Gauger, MA
}

For 10 years the Medical College of Wisconsin and Columbia St. Mary's Hospital have joined together in a partnership to work within some of Milwaukee's most impoverished neighborhoods. Beginning simply by providing health care through a free clinic, the partnership soon was confronted with numerous examples of barriers to care being experienced by patients. A community-based participatory action process allowed the local population to give voice to the local realities of barriers to care. Here we combine our anecdotal clinical experience, the neighborhood's input, and an example of a successful program from a low-resource international setting to create a novel approach to treating chronic disease in uninsured populations.

This model of care has been successful for 2 reasons. First, the model shows good health outcomes at low cost. Second, solid community partnerships with care providers, churches, and other groups have been formed in support of the model, ensuring its credibility and sustainability. ( $\mathrm{J}$ Am Board Fam Med 2013;26:311-315.)

Keywords: Chronic Disease, Community Medicine, Public Health

Milwaukee is home to 4 major health systems and several Fortune 500 companies, and it is ringed with some of the country's wealthiest counties. Yet despite the health and financial resources of the metro area, the city itself is deeply impoverished and sick-even more so than when the Medical College of Wisconsin (MCW) and Columbia St. Mary's (CSM) health care system began working collaboratively toward improving our community's health 10 years ago. When we started our efforts, poorer Milwaukeeans could still rely on a kind of local health insurance provided by the county government. But the Great Recession brought an end to that program, along with many jobs that once offered health benefits. As the city slouched its way

This article was externally peer reviewed.

Submitted 31 August 2012; revised 29 January 2013; accepted 4 February 2013.

From the Department of Family and Community Medicine, Medical College of Wisconsin, Milwaukee (JS); Columbia St. Mary's Hospital, Milwaukee, WI (BS); and Columbia St. Mary's Foundation, Milwaukee, WI (MG).

Funding: This research was supported by grants from the Healthiest Wisconsin Partnership Program (grant nos. 2007I-06, 2010I-07) and the Columbia St. Mary's Foundation.

Conflict of interest: none declared.

Corresponding author: James Sanders, MD, MPH, 1121 E. North Ave, Milwaukee, WI 53212 (E-mail: jsanders@mcw.edu). through the past decade, some key health indices worsened. For example, parts of Milwaukee have an infant mortality rate close to 20 per 1,000 -worse than Columbia, Bulgaria, and the Gaza Strip. ${ }^{1}$

Our institutions have responded to the city's needs with adaptive programming. This article is an account of the iteration of the programs, from the first cautious steps taken together, to the forging of trust and collective experience, to the risk-taking with new types of models of care, to the current expansion into new collaborative partnerships with communitybased groups.

\section{Laying the Foundations for Partnership}

The need for health care access is great in Milwaukee, where poverty was severe even before the economic crisis of 2008 caused a recession from which the country has been slow to recover. In 2010, the poverty rate in the United States was $15.2 \%$, but in Milwaukee-the fourth-poorest city in the nation, with $>171,000$ residents in poverty-the rate was $29.5 \%$, up from $27 \%$ in 2009 . Among minorities, the poverty rate was especially high: $41.4 \%$ among African Americans and 32.3\% among Hispanics. ${ }^{2}$ In the 2 most impoverished ZIP codes in Milwaukee, 53212 and 53206 (also the most prominent for 
patients in our community-based chronic disease management program [CCDM]), the unemployment rate for black men has approached $50 \%$, and at one point during the recession, there were 25 job seekers in the central city for every 1 available full-time job. ${ }^{3}$

Among African Americans, the striking differences in end-organ damage versus the US population at large are nothing less than a national tragedy. To wit, African Americans have a 33\% higher age-adjusted death rate for cardiovascular disease than the overall population and are almost twice as likely to have a first stroke than their white counterparts. ${ }^{4}$ Closer to home, in Wisconsin, diabetes kills twice as many African Americans as whites, stroke kills 50\% more African Americans than whites, and heart disease kills $20 \%$ more African Americans than whites. ${ }^{5}$

In 2002, CSM entered into an agreement with MCW to open a free clinic for the uninsured in Milwaukee that relied on physician volunteers. MCW provided students for their service-learning experience; CSM provided a facility and some supporting ancillary clinical services. For both institutions, the free-clinic experience has been very successful. For MCW, the opportunity to participate in this type of service-learning experience has been very popular and has resulted in the most subscribed to ancillary student activity in the school's history, with $>200$ student volunteers annually. For CSM, the clinic has helped it to meet its longheld mission of service to the poor and the community. For us, the authors, it has led to a durable community-academic partnership. ${ }^{6}$

\section{Barriers to Care}

Soon after beginning the free clinic, we formed a community-based participatory action initiative in nearby neighborhoods to address the social determinants affecting the health of some of our patients at the free clinic. ${ }^{7}$ Food scarcity, emotional stress and coping, community safety and security, and access to care were all subjects on which we collaborated to bring resources and programming to the community. Not long thereafter, we grew increasingly aware of a significant number of barriers to care that our patients with chronic disease were encountering regularly: financial costs associated with pharmaceuticals and monitoring supplies; lack of time to return to see a doctor; and lack of health education. These barriers meant that many of our patients could return only every 5 to 6 months for their care and, as a result, would be found to be "out of control" with regard to their chronic disease(s). Similarly, about the same time, CSM's parish nurses (who all hold a bachelors of science of nursing degree) were recognizing the same barriers as they worked in these neighborhoods. The nurses, who provided counsel, education, assistance in obtaining care, and health screenings, found many of their patients' diseases woefully out of control, with few clinical locations available for ongoing chronic disease care.

For us, it seemed that the best health outcomes were being achieved at the free clinic when patients presented with acute health problems that were discrete in their systemic involvement. When patients presented with chronic diseases such as hypertension, diabetes, or hypercholesterolemia, it was more difficult to achieve measurably good health outcomes because of the innumerable barriers to ongoing care with which the patients had to contend. This cyclical in control/out of control management of chronic diseases cried out for a model of care different from the office-based, doctor-centric approach that is the mainstay of much of American medicine.

Meanwhile, one of the authors (JS) became involved with a project addressing chronic disease in the impoverished Republic of Georgia. Because of extremely limited health-related resources and an overwhelming number of deaths directly attributable to uncontrolled hypertension, nurses there had been newly empowered to make clinical decisions with the aid of predesigned protocols. This approach freed the limited number of physicians to care for more complicated cases and to have time to supervise the nurses. As a result, costs dropped and hypertensive control markedly improved..$^{8-10}$

The experience in the Republic of Georgia made JS wonder about what could be done in Milwaukee. After all, many of the same barriers to care existed in both the Republic of Georgia and Milwaukee: financial hardship, no suitable transportation, lack of health education, etc. Another author (BS) also was wondering how to proceed, having heard from CSM parish nurses about several of their patients who had suffered strokes for want of timely and effective follow-up care.

\section{Venturing Forward Together}

Shortly thereafter, we began formulating a response to our neighborhood's need for improved 
chronic disease care in the uninsured population. We opened the CCDM in 2007 to care for people with certain types of chronic disease and access to effective care at a reasonable cost. The CCDM brought together proven models of chronic care in a novel setting - that of nurse-led teams using protocol-driven clinical decision making situated in 2 neighborhood food pantries. To date, our experience with the CCDM has demonstrated that such a model can achieve good outcomes. ${ }^{11}$

\section{Program Design}

We designed the CCDM to eliminate barriers to care so that patients could achieve high levels of treatment adherence despite having limited resources. The program did away with costly and burdensome elements of care (high-priced drugs, appointment systems, paper charts, etc.) and leveraged community-based and patient-centered resources (location, health care team leadership, culturally attuned education, etc.). Our care model was not intended to be a model for all patients with all diseases. Rather, the model concerned itself solely with 3 chronic diseases: high blood pressure (essential type), uncomplicated diabetes mellitus type 2, and hypercholesterolemia. Patients with other complaints or diseases would need to be referred elsewhere for ongoing care.

We placed the care sites in community settings so that many patients could reach them on foot or with simple public transportation. The hours of operation corresponded to those of the food pantry in which the clinics operated to facilitate one-stop convenience. Next, the human resource costs were kept down by having nurses lead the team effort and having nurse practitioners or physicians act only as consultants and supporters of the nurse-led efforts. In particular, parish nurses were used because of both their familiarity with the community and the community's familiarity with them. We developed clinical protocols to facilitate the nurses' independence and to get patients to their chronic disease goals expeditiously. The CCDM also was designed to help people enroll in state-funded insurance and refer people with Medicaid to federally qualified health centers.

In addition, we kept records on a secure web server so that any patient data could be accessed in real time from any location. We secured funding to allow for pharmaceuticals to be dispensed on site and for laboratory phlebotomy to be offered on site as well. Funding was provided by the CSM Foundation and the Healthier Wisconsin Partnership Program (HWPP) of the Medical College of Wisconsin, but much of the cost (rent, utilities, furnishings, etc.) was covered through in-kind donations from CCDM's community partners.

The CCDM's emphasis on community-based services has meant that outreach into underserved neighborhoods has been all the easier to initiate, and the lower comparable costs of the CCDM has meant that these services could be sustained more easily, especially if the higher costs associated with the long-term disabilities from uncontrolled chronic disease are considered.

\section{Development of the Health Care Workforce}

Resident physicians and nursing students come to the CCDM for precepted service-learning experiences. For the resident physician, it offers a valuable vehicle for understanding economic and social factors that shape health as well as inclusive and apt ways to address patient needs. The Institute of Medicine recognized this very type of experience when it called on American medical school curricula to address social inequalities and determinants of health. ${ }^{12}$ Senior nursing students from the University of Wisconsin-Milwaukee use the CCDM in a similar manner. At the CCDM, they personally experience community health at its core, in the real world beyond their textbooks. They learn firsthand why impoverished people do not have health insurance, and they learn that many vulnerable patients are unaware of how their behavioral choices influence overall health. One of the authors (BS) developed a presentation, titled "What I Have Learned About People and Poverty," to provide a new perspective to medical students, residents, and nursing students in preparation for their respectful service to CCDM patients. In addition, a booklet of patient stories from the first 3 years of service was produced to show that the CCDM served a variety of people and to debunk myths about the nature of people in poverty.

But perhaps the most novel way by which the CCDM has contributed to workforce development has been its network involving parish nurses, community health workers, and volunteer health liaisons from local Church of God in Christ (COGIC) churches. The liaisons serve as bridges between members of partnering churches and CCDM; under the nurses' oversight, the community health 
workers collaborate with the liaisons to be sure that needed preventive education, screening, and CCDM services are offered. ${ }^{13}$ The CCDM thus has tapped into the community it serves and expanded clinical care effectively. Studies indicate that church collaborations tailored to deal with particular health concerns are valuable, finding that because churches are "vital and influential" for African Americans, alliances of faith-based institutions, public health authorities, academics, and voluntary organizations are opening windows for "needed health information and culturally appropriate interventions intended to eliminate health disparities." 14 The community health workers have enhanced access to care, promoted proper use of health resources, and furnished cultural links between communities and delivery systems. ${ }^{15} \mathrm{We}$ learned firsthand about the value of these linkages after a church elder announced that she had breast cancer. The liaison asked parish nurses to devote extra attention to breast cancer awareness and mammography during the month after the announcement, when women in the church became invested in the issue; the liaison then helped uninsured women to obtain coverage for mammography.

\section{Forming Partnerships}

A challenge for many organizations working to improve community health is to sustain their programming for years. The CCDM likewise faced the challenge of improving the treatment of chronic diseases for many years, not just for a grant cycle. Knowing that strong partnerships are vital to sustainability, the CCDM developed partnerships with CSM community programs and the COGIC Covenant, as well as with MCW. Such collaboration allows resources to be provided and shared to sustain the quality of care and community presence that are essential to the CCDM.

MCW is a partner on 2 levels. On one level, it is the academic home of one author (JS), whose role as an MCW faculty member at the CSM family medicine residency positions him as a community provider as well as a mentor for residents interested in primary care practice in an urban setting. His belief that community health is critical to personal health informs programs such as the CCDM. Another level of MCW partnership is through the HWPP, which supports community health through community-
MCW partnerships. An HWPP Request for Proposals brought the authors together to propose the initial CCDM model, which HWPP funded. A later, longer cycle of Healthier Wisconsin funding allowed the CCDM to expand to multiple sites.

CSM also is a partner on 2 levels. First, it provides multiple community-based programs that collaborate with the CCDM, among them St. Elizabeth Ann Seton Dental Clinic, the urgent care dental provider for the poor in Milwaukee. Seton requires patients to have safe blood pressure levels before urgent care, which often involves extraction of damaged teeth, raising stress and blood pressure levels. Many of the clinic's uninsured patients have high blood pressure and lack a primary care provider who can help them gain control of it. They are referred to the CCDM, which helps them establish control of their blood pressure, begin managing their chronic disease, and in turn receive the dental care they need.

Second, CSM employs the parish nurses who conducted the original outreach to the food pantries and who serve as the CCDM clinic nurses. We have found that those patients whom the nurses discovered in church outreach work are extremely happy to see the nurses again in the CCDM. Having established longstanding relationships in the communitychurches and agencies such as literacy programs, senior centers, and outreach services-the nurses report that they have the trust of patients who have been socially marginalized and often otherwise have misgivings about health care professionals and health systems.

The COGIC Covenant was the outgrowth of parish nurses' work with churches that serve primarily African American members in Milwaukee. CSM nurses had collaborated with COGIC churches in health education and screening so successfully that Bishop C. H. McClelland asked them to coordinate the health ministry for the 45 churches in his jurisdiction. One author (BS) and McClelland then signed a covenant to work together to improve the health of congregants and the community at large. The oversight and skills of the parish nurses are essential to the COGIC Covenant model of health outreach, which aims to leave no congregant undiagnosed or untreated for hypertension and diabetes. Each CCDM clinic is hosted by a COGIC church, a further illustration of partnership strength.

Finally, the CCDM amplifies its community presence through its role in the Free and Commu- 
nity Clinic Collaborative. The Milwaukee group includes medical and dental providers that provide care free or at low cost, largely to uninsured people. Many of the clinics provide only episodic care, so they appreciate the ability to make referrals to the CCDM for long-term management of diabetes and hypertension. In turn, the clinics, which often provide care for health problems that the CCDM does not treat, accept referrals from the CCDM for patients with, for example, respiratory or orthopedic problems.

\section{Conclusion}

The ill health of Milwaukee is linked to its deep poverty. Our efforts over the past 10 years have aimed to break this link by providing health care. We have shown that a novel approach can have good outcomes with low costs. By breaking down barriers to care, we have demonstrated success with a sustainable community-based model for addressing common chronic diseases that if left unchecked would lead to many severe health disparities. The communities themselves are being empowered through our efforts with them, and in turn are surely building toward improved health in the broadest sense.

The CCDM clinical staff includes Brenda Buchanan, RN; Julia Means, RN; Ebonie Gray, APNP; and Heather Puente, MPH. The family medicine residents from the Columbia St. Mary's Family Medicine Residency Program are thanked for their consultative services.

\section{References}

1. Stephenson C. For Milwaukee's children, an early grave. Milwaukee Journal Sentinel, September 22, 2011. Available from http://www.jsonline.com/news/ milwaukee/114430774.html. Accessed August 28, 2012.

2. Tolan T, Herzog K. Poverty numbers spike in Milwaukee. Milwaukee Journal Sentinel, September 21, 2011. Available from http://www.jsonline.com/news/ milwaukee/Poverty-numbers-spike-in-Milwaukee.html. Accessed July 27, 2012.

3. Pawasarat J, Quinn LM, Bruecker R, Lohman E, Schumann N, Vitkovic S, Regional Workforce Alliance. Survey of job openings in the 7 counties of Southeastern
Wisconsin: week of May 25, 2009. Available from http:// www4.uwm.edu/eti/2009/RegionalJobOpenings.pdf. Accessed August 13, 2012.

4. Lloyd-Jones D, Adams R, Carnethon M, et al; American Heart Association Statistics Committee and Stroke Statistics Subcommittee. Heart disease and stroke statistics-2009 update: a report from the American Heart Association Statistics Committee and Stroke Statistics Subcommittee. Circulation 2009;119: e21-181.

5. Henry J. Kaiser Family Foundation. Statehealthfacts.org [homepage]. Available from http://www.statehealthfacts. org. Accessed March 13, 2013.

6. Wolff M, Maurana C. Building effective community-academic partnerships to improve health: a qualitative study of perspectives from communities. Acad Med 2001;76:166-72.

7. Sanders J, Baisch MJ. Community-based participatory action: impact on a neighborhood level community health improvement process. Prog Community Health Partnersh 2008;2:7-15.

8. Grim CE, Grim CM, Peterson JR, et al. Prevalence of cardiovascular risk factors in the Republic of Georgia. J Hum Hypertens 1999;13:243-7.

9. Sanders J. A family medicine training program in the Republic of Georgia - incorporating a model of chronic disease management. J Am Board Fam Med 2007;20:557-64.

10. Barbakadze VT, Koblianidze LG, Kipshidze NN, Grim CE, Crim CM, Tavill F. The Republic of Georgia High Blood Pressure Control Program. Ethn Dis 2006;16:61-5.

11. Sanders J, Guse C, Onuoha B. Pilot study of a new model for managing hypertension in an uninsured population. J Prim Care Community Health. 2013; 4:44-9.

12. Doran KM, Kirley K, Barnosky AP, Williams JC, Cheng JE. Developing a novel poverty in healthcare curriculum for medical students at the University Of Michigan Medical School. Acad Med 2008;83:5-13.

13. Solberg B. Health liaisons expand the power of parish nurses. Health Progr 2013;94:55-7.

14. Airhihenbuwa C, Liburd L. Eliminating health disparities in the African American population: the interface of culture, gender, and power. Health Educ Behav 2006;33:488-501.

15. Witmer A, Seifer S, Finocchio L, Leslie J, O’Neil E. Community health workers: integral members of the healthcare workforce. Am J Public Health 1995;85(8 Pt 1):1055-8. 